\title{
Prognostic significance of the co-expression of nucleophosmin and trefoil factor 3 in postoperative gastric cancer patients
}

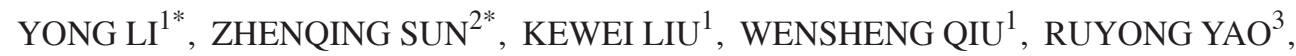 \\ TONGTONG FENG ${ }^{1}, \mathrm{CHAO}^{\mathrm{XIN}}{ }^{1}$ and $\mathrm{LU} \mathrm{YUE}^{1}$ \\ Departments of ${ }^{1}$ Oncology and Tumor Molecular and Translational Medicine Laboratory and ${ }^{2}$ General Surgery; \\ ${ }^{3}$ Central Laboratory, The Affiliated Hospital of Qingdao University Medical College, \\ Qingdao, Shandong 266003, P.R. China
}

Received May 22, 2014; Accepted July 7, 2014

DOI: $10.3892 / \mathrm{mco} .2014 .351$

\begin{abstract}
Although a number of studies have indicated that the positive expression of nucleophosmin (NPM) and trefoil factor 3 (TFF3) is associated with oncogenesis and poor prognosis in several tumor types, the prognostic value of the co-expression of NPM and TFF3 in gastric cancer (GC) has not been fully elucidated. Therefore, in this study, we aimed to investigate the role of NPM and TFF3 in GC and determine their prognostic value. We retrospectively reviewed 108 patients who had undergone radical gastric tumor resection. The expression of NPM and TFF3 was detected by immunohistochemistry and the association of NPM and TFF3 with clinicopathological characteristics was investigated using the Chi-square test. Furthermore, univariate and multivariate analyses were conducted to determine the prognostic value of these markers. Of the 108 samples, NPM was positive in $57(53 \%)$ and TFF3 was positive in 54 samples (50\%). The positive expression of NPM was correlated with advanced tumor stage and recurrence $(\mathrm{P}=0.0333$ and $\mathrm{P}<0.0001$, respectively), whereas the expression of TFF3 was associated with larger tumor size $(\mathrm{P}=0.0005)$, poor differentiation $(\mathrm{P}=0.0435)$, lymph node metastasis $(\mathrm{P}=0.0116)$, advanced tumor stage $(\mathrm{P}=0.0244)$ and recurrence $(\mathrm{P}=0.0116)$. The univariate analysis revealed that the expression of NPM, the expression of TFF3 and the co-expression of the two were associated with poor survival $(\mathrm{P}=0.0004,0.0028$ and 0.0020 , respectively). By multivariate analysis, all three factors were identified as independent prognostic factors in postoperative GC patients (hazard ratio $=1.970,2.021$ and 2.339 , respectively). In conclusion,
\end{abstract}

Correspondence to: Dr Lu Yue, Department of Oncology and Tumor Molecular and Translational Medicine Laboratory, The Affiliated Hospital of Qingdao University Medical College, 16 Jiangsu Road, Qingdao, Shandong 266003, P.R. China

E-mail: qdyuelu@163.com

*Contributed equally

Key words: gastric neoplasm, nucleophosmin, trefoil factor 3, prognosis, biomarkers the expression of NPM and TFF3 and, particularly, the co-expression of the two, may serve as independent prognostic factors in postoperative GC patients.

\section{Introduction}

Gastric cancer (GC) is the third most common malignancy and the second leading cause of cancer-related mortality worldwide, particularly in East Asian countries. In China, the majority of the GC patients are diagnosed at an advanced stage and, despite the advances in chemotherapy and surgical techniques, the 5-year overall survival rate of patients with advanced-stage GC is $<20 \%$ (1-2). In GC cases, physicians are faced with the following challenges: lack of markers for early diagnosis, weak prognostic significance of histological indicators, limited efficiency of current treatment for advanced GC and lack of molecular markers for targeted therapy (3-5). Therefore, it is of great clinical significance to achieve a better understanding of gastric carcinogenesis and to identify novel markers for the improvement of clinical management of patients with GC.

Nucleophosmin (NPM), also referred to as protein B23, numatrin and NO38, is a nucleolar phosphoprotein (6). As a multifunctional protein, NPM participates in ribosome biosynthesis and centrosome duplication and also acts as a molecular chaperone, promoting cell proliferation and regulating apoptosis via the nuclear factor (NF)- $\mathrm{B}$ pathway (7-8). Positive expression of NPM has been identified in several tumor types, including colorectal adenoma (9), hepatocellular carcinoma (10), prostate cancer (11), bladder cancer (12), ovarian cancer (13) and bronchial tumors (14). As regards GC, Tanaka et al (15) reported that advanced GC appears to exhibit higher NPM mRNA levels. However, the protein expression and prognostic value of NPM in GC has not been extensively investigated.

Trefoil factor 3 (TFF3), also referred to as intestinal trefoil factor, is a member of the trefoil peptide family. TFF3 is mainly secreted by goblet cells in the small and large intestine. There is almost no TFF3 expression in normal gastric mucosa. However, TFF3 expression may be detected in cases with intestinalization or GC (16-17). It was previously demonstrated that TFF3 may inhibit cell adhesion and promote cell invasion 
by downregulating E-adhesin expression (18), as well as blocking apoptosis through the NF- $\mathrm{KB}$ signaling pathway (19). Previous studies also reported that the positive expression of TFF3 was correlated with a poor prognosis (20-21).

Based on the abovementioned knowledge, we hypothesized that NPM and TFF3 played a key role in carcinogenesis. It was recently demonstrated that there is a close association of human epidermal growth factor receptor 2 with NPM and TFF3 expression and that their co-expression may play a pivotal role in GC (22-23). Furthermore, there is evidence demonstrating that NPM and TFF3 are involved in the regulation of apoptosis via the $\mathrm{NF}-\kappa \mathrm{B}$ signaling pathway, prompting us to determine the significance of their co-expression in GC. Therefore, in the present study, we investigated the expression of NPM and TFF3 in GC tissues and analyzed their correlation with clinicopathological variables and prognosis.

\section{Patients and methods}

Patients and samples. GC and adjacent tissues were obtained from patients who underwent radical gastrectomy at the Affiliated Hospital of Qingdao University Medical College between November, 2007 and March, 2009. All the patients met the following criteria: i) All the tumors had been histologically confirmed as adenocarcinomas; ii) no patients had received preoperative chemotherapy or radiotherapy; and iii) all the patients were available for follow-up. Finally, 108 patients were enrolled in this study. Clinicopathological information, including gender, age ( $<60$ vs. $\geq 60$ years), tumor size ( $\leq 4 \mathrm{vs} .>4 \mathrm{~cm}$ ), tumor differentiation (high, moderate or poor), lymph node metastasis, tumor stage and primary vs. recurrent tumor, were obtained from the patients' medical records and are summarized in Table I. The tumor-node-metastasis (TNM) stage was assessed according to the 7th edition of the TNM Classification of Malignant Tumors (24). Stages I and II were classified as early, whereas stages III and IV were classified as advanced. The median duration of follow-up was 31 months (range, 3-53 months). For patients who remained alive until the cut-off date of follow-up, survival duration was recorded as $53^{+}$months.

The study protocol was approved by the Ethics Committee of the Affiliated Hospital of Qingdao University Medical College and informed consent was obtained from all the patients prior to enrolment.

Immunohistochemistry. Paraffin-embedded tissue blocks were cut in $4-\mu \mathrm{m}$ sections. Following dewaxing and rehydration, the slides were incubated in peroxidase-blocking solution to block endogenous peroxidase activity. For antigen retrieval, all the sections were incubated in citrate buffer solution ( $\mathrm{pH}$ 6.0) in a microwave oven for $20 \mathrm{~min}$. Subsequently, the specimens were incubated at $37^{\circ} \mathrm{C}$ for $90 \mathrm{~min}$ in anti-NPM mouse monoclonal antibody (dilution, 1:100; Abcam, Cambridge, MA, USA) or in TFF3 monoclonal mouse antibody (dilution, 1:100; Abcam). The sections were then incubated with reagent 1 and 2 of the PV9005 mouse hypersensitivity two-step immunohistochemical kit (Beijing fir Jinqiao, Beijing, China) for a total duration of $60 \mathrm{~min}$ at $37^{\circ} \mathrm{C}$ in a humid chamber. Finally, the staining was visualized with diaminobenzidine. The slides were counterstained with hematoxylin, washed in tap water for $10 \mathrm{~min}$ and then mounted. Between each pair of steps, the sections were rinsed with phosphate-buffered saline (PBS). A negative control reaction was set with PBS replacing the anti-NPM or anti-TFF antibody, while known positive-stained sections were used as positive control.

Scoring of immunostaining. Each slide was scored by two experienced pathologists who were blinded to the clinical outcome. Immunohistochemical staining was assessed semiquantitatively by measuring the extent of staining $(0,0 \%$; $1,0-10 \% ; 2,10-50 \%$; and $3,50-100 \%$ ) as well as the intensity of staining ( 0 , no staining; 1 , yellow; 2 , brown-to-yellow; and 3 , brown staining). The weighted score for each case according to the intensity and extent of staining were multiplied $[0$, negative (-); $1-4$, weakly positive $(+) ; 5-8$, moderately positive $(++)$; and $9-12$, strongly positive $(+++)]$. The weighted scores of $0-4$ were considered as negative and 5-12 as positive.

Statistical analysis. The Chi-square test was used to analyze the association between the expression of NPM and TFF3 and clinicopathological characteristics. Survival curves were plotted with the Kaplan-Meier method. The significance of the difference between groups was assessed with the log-rank test. Multivariate analyses (Cox proportional hazard regression models) were performed to assess the prognostic value of NPM and TFF3. The clinical variables included gender, age, tumor size, differentiation, lymph node metastasis and stage. SAS 9.2 software (SAS Institute, Inc., Cary, NC, USA) was used for statistical analysis. $\mathrm{P} \leq 0.05$ was considered to indicate a statistically significant difference.

\section{Results}

NPM and TFF3 immunohistochemical staining. NPM was mainly expressed in the nucleoli, nuclei and cytoplasm of tumor epithelial cells (Fig. 1A and B) and TFF3 was mainly expressed in the cytoplasm (Fig. 1C and D). Of the 108 specimens, the positive expression rates of NPM in neoplastic tissue and adjacent gastric mucosa were 53 and $37 \%(\mathrm{P}<0.05)$, respectively. Of the 57 NPM-positive neoplastic tissue specimens, 25 were TFF3-positive and 32 TFF3-negative. Of the 54 TFF3-positive neoplastic tissue specimens (50\%), 25 were NPM-positive and 29 NPM-negative (Table II). No moderate or strong positive expression was identified in the adjacent gastric mucosa. By correlation analysis, no significant association was found between the expression of NPM and TFF3 $(\mathrm{r}=0.11119, \mathrm{P}=0.2520)$.

Correlation between NPM, TFF3 and clinicopathological characteristics. We analyzed the associations between NPM and TFF3 expression and clinical characteristics (Table I) and demonstrated that NPM-positive expression was correlated with advanced tumor stage $(\mathrm{P}=0.0333)$. Of the 80 cases with primary tumor, 33 exhibited positive staining for NPM, whereas of the 28 cases with recurrence, 24 exhibited positive staining for NPM, suggesting that NPM was associated with recurrence $(\mathrm{P}<0.0001)$. There was no statistically significant association between NPM expression and age $(\mathrm{P}=0.5020)$, gender $(\mathrm{P}=0.1891)$, tumor size $(\mathrm{P}=0.4343)$ or differentiation $(\mathrm{P}=0.5652)$. 
Table I. NPM or TFF3 staining and clinicopathological characteristics.

\begin{tabular}{|c|c|c|c|c|c|c|c|c|c|c|}
\hline \multirow[b]{2}{*}{ Characteristics } & \multirow[b]{2}{*}{$\begin{array}{c}\text { Cases } \\
(n=108)\end{array}$} & \multicolumn{2}{|c|}{ NPM staining } & \multirow[b]{2}{*}{ P-value } & \multicolumn{2}{|c|}{ TFF3 staining } & \multirow[b]{2}{*}{ P-value } & \multicolumn{2}{|c|}{$\begin{array}{c}\text { Co-expression } \\
\text { of NPM and TFF3 }\end{array}$} & \multirow[b]{2}{*}{ P-value } \\
\hline & & $\begin{array}{l}\text { Positive } \\
(\mathrm{n}=57)\end{array}$ & $\begin{array}{l}\text { Negative } \\
(n=51)\end{array}$ & & $\begin{array}{l}\text { Positive } \\
(\mathrm{n}=54)\end{array}$ & $\begin{array}{l}\text { Negative } \\
(n=54)\end{array}$ & & $\begin{array}{l}\text { Positive } \\
(\mathrm{n}=25)\end{array}$ & $\begin{array}{l}\text { Negative } \\
(n=83)\end{array}$ & \\
\hline Gender & & & & 0.1891 & & & 0.3993 & & & 0.4262 \\
\hline Male & 76 & 37 & 39 & & 36 & 40 & & 16 & 60 & \\
\hline Female & 32 & 20 & 12 & & 18 & 14 & & 9 & 23 & \\
\hline Age (years) & & & & 0.5020 & & & 0.0195 & & & 0.7649 \\
\hline$<60$ & 46 & 26 & 20 & & 17 & 29 & & 10 & 36 & \\
\hline$\geq 60$ & 62 & 31 & 31 & & 37 & 25 & & 15 & 47 & \\
\hline Tumor size $(\mathrm{cm})$ & & & & 0.4343 & & & 0.0005 & & & 0.0002 \\
\hline$\leq 4$ & 53 & 30 & 23 & & 17 & 36 & & 4 & 49 & \\
\hline$>4$ & 55 & 27 & 28 & & 32 & 22 & & 21 & 34 & \\
\hline Differentiation & & & & 0.5652 & & & 0.0435 & & & 0.1529 \\
\hline High & 5 & 2 & 3 & & 1 & 4 & & 0 & 5 & \\
\hline Moderate & 10 & 4 & 6 & & 2 & 8 & & 1 & 9 & \\
\hline Poor & 93 & 51 & 42 & & 51 & 42 & & 25 & 58 & \\
\hline Lymph node metast & asis & & & 0.2754 & & & 0.0116 & & & 0.0156 \\
\hline Present & 61 & 35 & 26 & & 37 & 24 & & 23 & 38 & \\
\hline Absent & 47 & 22 & 25 & & 17 & 30 & & 2 & 45 & \\
\hline Tumor stage & & & & 0.0333 & & & 0.0244 & & & 0.0320 \\
\hline Early & 26 & 9 & 17 & & 8 & 18 & & 2 & 24 & \\
\hline Advanced & 82 & 48 & 34 & & 46 & 36 & & 23 & 59 & \\
\hline Primary/recurrence & & & & $<0.0001$ & & & 0.0116 & & & $<0.0001$ \\
\hline Primary tumor & 80 & 33 & 47 & & 33 & 47 & & 10 & 70 & \\
\hline Recurrence tumor & 28 & 24 & 4 & & 21 & 7 & & 15 & 13 & \\
\hline
\end{tabular}

NPM, nucleophosmin; TFF3, trefoil factor 3.

Table II. Expression of NPM and TFF3.

\begin{tabular}{lccr}
\hline & \multicolumn{2}{c}{ NPM expression } & \\
\cline { 2 - 3 } $\begin{array}{l}\text { TFF3 } \\
\text { expression }\end{array}$ & Positive & Negative & Total \\
\hline Positive & 25 & 29 & 54 \\
Negative & 32 & 22 & 54 \\
Total & 57 & 51 & 108
\end{tabular}

$\mathrm{r}=0.11119, \mathrm{P}=0.2520 . \mathrm{NPM}$, nucleophosmin; TFF3, trefoil factor 3.

As regards TFF3, there were significant correlations between TFF3-positive expression and advanced age $(\mathrm{P}=0.0195)$, larger tumor size $(\mathrm{P}=0.0005)$ and poor differentiation $(\mathrm{P}=0.0435)$, whereas, by further pairwise comparison, a significant difference was found between moderate and poor differentiation ( $\mathrm{P}=0.0312$, data not shown), lymph node metastasis $(\mathrm{P}=0.0116)$, advanced tumor stage $(\mathrm{P}=0.0244)$ and recurrence $(\mathrm{P}=0.0116)$. There was no significant association between TTF3 and gender $(\mathrm{P}=0.3993)$.
The co-expression of NPM and TFF3 was associated with large tumor size $(\mathrm{P}=0.0002)$, lymph node metastasis $(\mathrm{P}=0.0156)$, advanced tumor stage $(\mathrm{P}=0.0320)$ and recurrence $(\mathrm{P}<0.0001)$. There was no significant association between the co-expression of these two factors and other clinicopathological parameters.

Prognostic significance of NPM and TFF3. To assess the prognostic significance of NPM and TFF3, Kaplan-Meier survival curves were constructed. A statistically significant correlation was observed between NPM-positive expression and poor survival ( $\mathrm{P}=0.0004$, log-rank test, Fig. 2A). TFF3-positive expression was associated with a poor survival rate compared to TFF3-negative expression ( $\mathrm{P}=0.0028$, log-rank test, Fig. $2 \mathrm{~B}$ ). Patients with co-expression of NPM and TFF3 exhibited lower survival rates compared to patients without co-expression of the two factors $(\mathrm{P}=0.0020$, log-rank test, Fig. 2C).

We also divided the samples into 4 subgroups based on the different expressions of NPM and TFF3. NPM-negative and TFF3-negative was designated as group 1; NPM-negative and TFF3-positive was designated as group 2; NPM-positive and TFF3-negative was designated as group 3; and NPM-positive and TFF3-positive was designated as group 4. The survival 

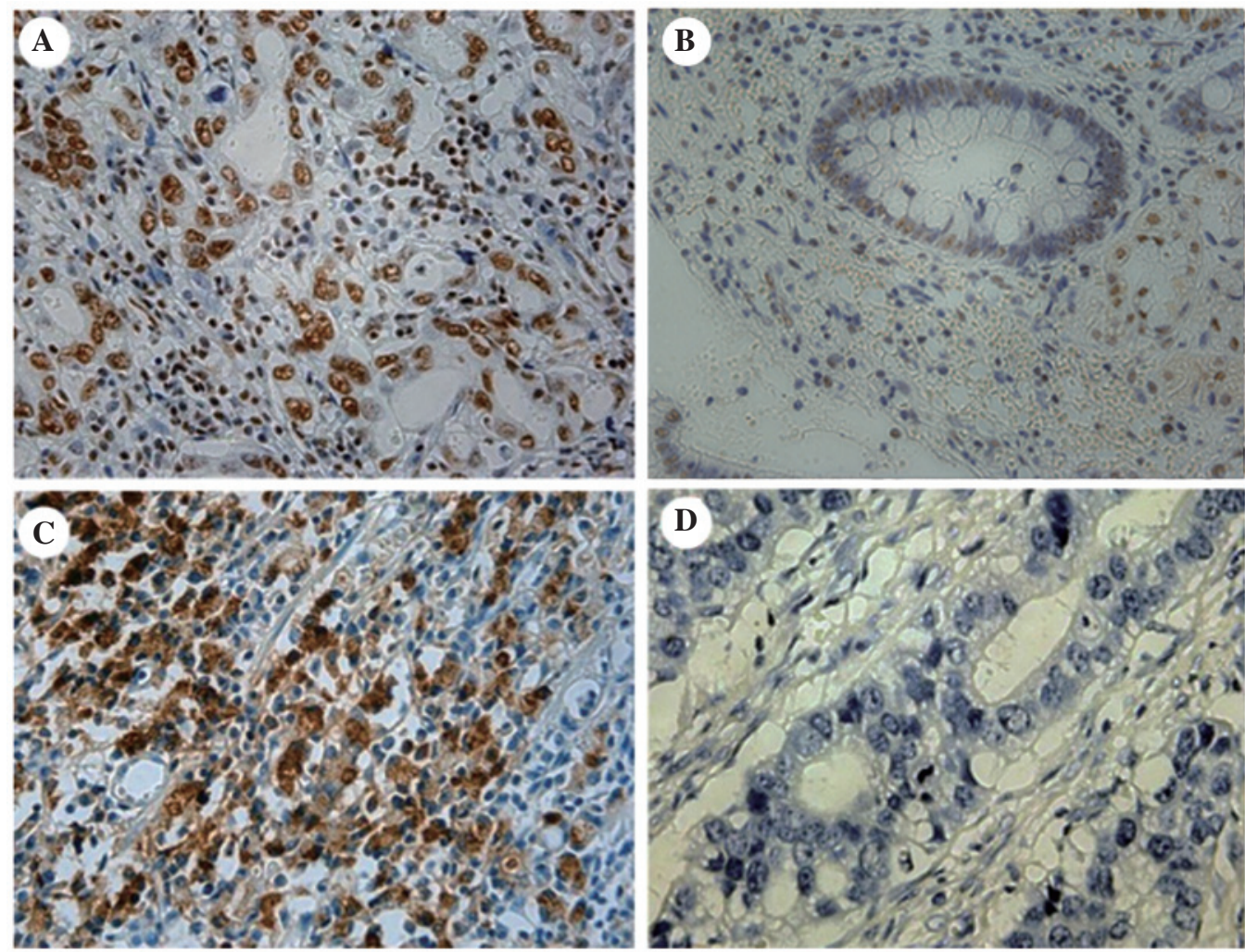

Figure 1. Immunohistochemical analysis of nucleophosmin (NPM) and trefoil factor 3 (TFF3) expression in gastric cancer tissue samples (magnification, $\mathrm{x} 400$ ). (A) NPM-positive staining [(++) and (+++)], mainly expressed in the nucleoli, nuclei and cytoplasm of tumor cells. (B) NPM-negative staining [(-) and (+)]. (C) TFF3-positive staining, mainly expressed in the cytoplasm. (D) TFF3-negative staining.

A

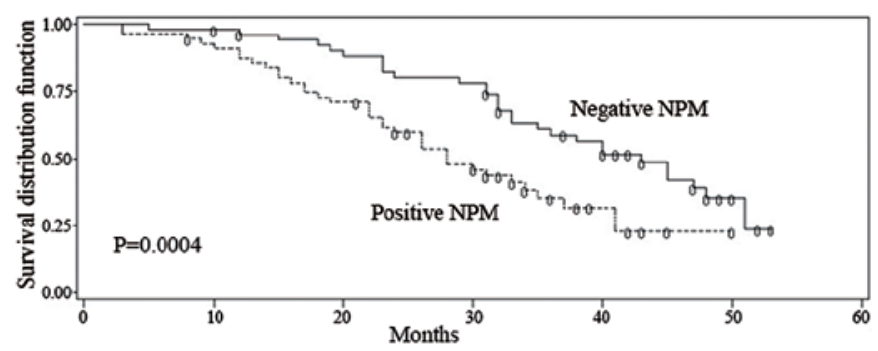

C

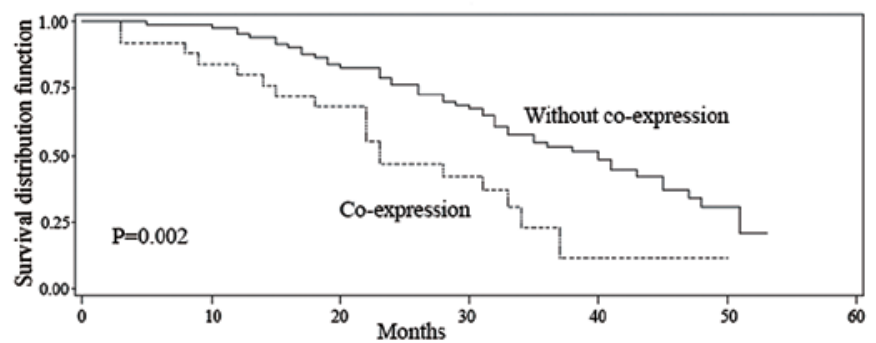

B

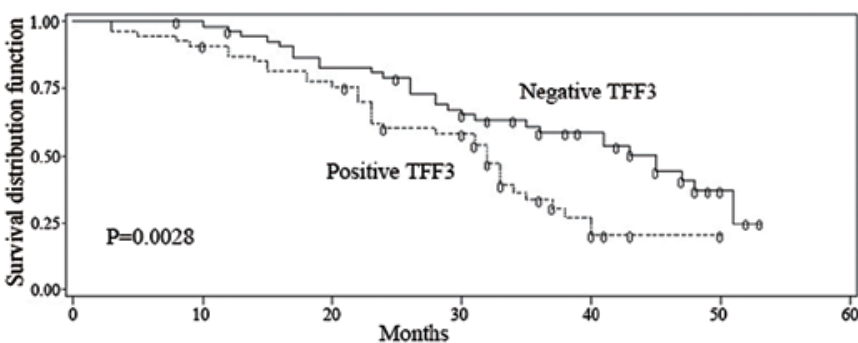

D

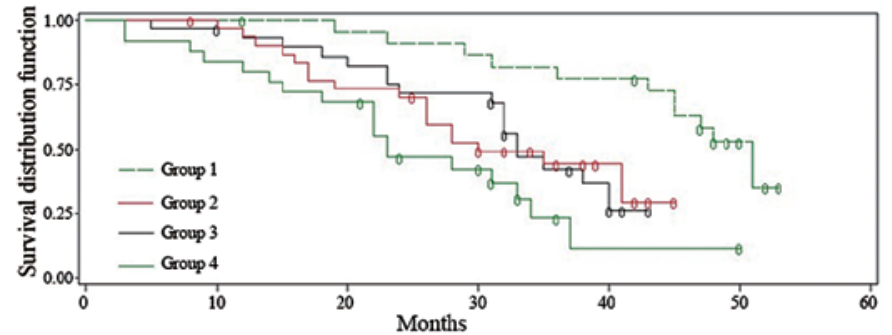

Figure 2. Kaplan-Meier estimates of the overall survival rate according to (A) the expression of nucleophosmin (NPM) (P=0.0004, log-rank test); (B) the expression of trefoil factor 3 (TFF3) ( $\mathrm{P}=0.0028$, log-rank test). (C) the co-expression of NPM and TFF3 ( $\mathrm{P}=0.0020$, log-rank test). (D) Survival curve of the 4 groups. Group 1, NPM-negative and TFF3-negative (green curve, interrupted line); group 2, NPM-negative and TFF3-positive (red curve); group 3, NPM-positive and TFF3-negative (black curve); group 4, NPM-positive and TFF3-positive (green curve, continuous line).

curves indicated that patients with co-expression of NPM and TFF3 exhibited the lowest survival rate when compared to the other 3 groups (Fig. 2D). Correspondingly, patients with negative expression for both markers exhibited the highest survival rate, while patients expressing either NPM or TFF3 exhibited intermediate survival rates (Fig. 2D). The detailed survival data of the 4 subgroups are described in Table III.

The univariate analysis revealed that NPM-positive expression, TFF3-positive expression, co-expression of NPM and TFF3, age, tumor stage and lymph node metastasis were 
Table III. Survival data of 4 subgroups.

\begin{tabular}{|c|c|c|c|c|c|c|}
\hline \multirow[b]{2}{*}{ Subgroups } & \multirow{2}{*}{$\begin{array}{l}\text { Median } \\
\text { survival time } \\
\text { (months) }\end{array}$} & \multirow{2}{*}{$\begin{array}{l}\text { Survival time } \\
\text { interval } \\
\text { (months) }\end{array}$} & \multicolumn{4}{|c|}{ Survival rate } \\
\hline & & & 12 months & 24 months & 36 months & 48 months \\
\hline NMP-, TFF3- & 39 & $12-53^{+}$ & 1.0000 & 0.8696 & 0.6857 & 0.3532 \\
\hline NPM-, TFF3+ & 32 & $5-43^{+}$ & 0.9298 & 0.7152 & 0.4197 & 0.3257 \\
\hline NPM+, TFF3- & 28 & $8-45^{+}$ & 0.9333 & 0.7000 & 0.4410 & N/A \\
\hline NPM+, TFF3+ & 23 & $3-50^{+}$ & 0.8000 & 0.5100 & 0.2119 & 0.2119 \\
\hline
\end{tabular}

NPM, nucleophosmin; TFF3, trefoil factor 3; N/A, not available.

Table IV. Univariate and multivariate survival analysis.

\begin{tabular}{lccccc}
\hline & $\begin{array}{c}\text { Univariate analysis } \\
\text { P-value } \\
\text { Parameters }\end{array}$ & \multicolumn{3}{c}{ Cox regression survival analysis } \\
\cline { 3 - 6 } (Log-rank test) & Chi-square & P-value & Hazard ratio & 95\% CI \\
\hline NPM-positive expression & 0.0004 & 57.849 & 0.0162 & 1.970 & $1.134-3.422$ \\
TFF3-positive expression & 0.0028 & 70.962 & 0.0077 & 2.021 & $1.204-3.391$ \\
Co-expression of NPM and TFF3 & 0.0020 & 88.712 & 0.0029 & 2.339 & $1.337-4.092$ \\
Age & 0.0261 & 44.381 & 0.0351 & 1.765 & $1.040-2.994$ \\
Tumor stage & 0.0004 & 174.061 & $<.0001$ & 5.539 & $2.478-12.379$ \\
Tumor size & 0.2028 & 13.231 & 0.2500 & 1.351 & $0.809-2.257$ \\
Differentiation & 0.7221 & 0.5422 & 0.8775 & 1.056 & $0.530-2.103$ \\
Lymph node metastasis & 0.0356 & 94.943 & 0.0021 & 2.604 & $1.417-4.787$ \\
\hline
\end{tabular}

95\% CIs, 95\% confidence intervals; NPM, nucleophosmin; TFF3, trefoil factor 3.

significantly associated with poor prognosis. By Cox regression survival analysis, NPM-positive expression, TFF3-positive expression, co-expression of NPM and TFF3, age and tumor stage were identified as independent prognostic factors in postoperative GC patients (Table IV).

\section{Discussion}

Focusing on different molecular markers may help us understand the histopathological characteristics of GC by detecting the expression of different and specific genes in GC tissues. In our study, we demonstrated that NPM overexpression was an independent prognostic factor in postoperative GC patients. In particular, we demonstrated that the co-expression of NPM and TFF3 was associated with a more aggressive biological behavior and poor prognosis in GC patients.

NPM, the focus of several recent cancer studies, plays a critical role in the development and progression of malignant tumors. Although evidence demonstrates that NPM expression is correlated with unfavorable clinical characteristics and poor prognosis in several types of solid tumors, the precise role of NPM in GC has not been clearly determined. In the present study, we observed that the positive expression of NPM was mainly localized to the nucleus and cytoplasm in GC cells, consistent with a previous study reporting that NPM shuttled between the nucleus and cytoplasm in other tumor types (6). It was previously demonstrated that a higher NPM mRNA expression was detected in gastric tumors (12). In this study, we detected NPM expression at the protein level and found that a positive expression of NPM was inversely correlated with prognosis in patients who underwent radical gastric resection. Moreover, in the present study, we observed that NPM was associated with lymph node metastasis and tumor recurrence, which was consistent with previous findings that reported NPM overexpression in $73 \%$ of GC patients with disease recurrence (25). Therefore, downregulating the level of NPM expression in GC may decrease the recurrence rate. Interestingly, we did not observe any significant differences in NPM expression between well-differentiated and poorly differentiated tumors, while Tsui et al (26) reported that NPM was also associated with differentiation in bladder tumors. This discordance may due to the different histological type (the GC type was adenocarcinoma, whereas the bladder carcinoma was of the transitional cell type). Based on this difference, it is reasonable to hypothesize that NPM may have different functions in different histological tumor types. However, as the number of studies focusing on NPM in gastric tumors is limited, the mechanism of NPM in gastric tumorigenesis has not been clearly determined and further studies are required to elucidate it. 
As regards TFF3, we observed that the positive expression of TTF3 was associated with tumor size, differentiation, lymph node metastasis and tumor stage (Table I), suggesting that TFF3 may play an important role in the development, progression and dissemination of GC. Recently, Meng et al (27) demonstrated that the positive expression of TFF3 was significantly associated with a lower survival rate in GC compared to negative expression; however, in that study, a multivariate analysis was not performed. In the present study, by multivariate analysis, we confirmed that the positive expression of TFF3 was an independent prognostic indicator, consistent with the findings of previous studies $(20,21)$. However, Dhar et al (28) reported that TFF3 expression was prognostically significant only in female patients, but not in the overall patient population. In our study, we conducted an additional survival analysis separately for female, male and overall patients and demonstrated that TFF3 was of prognostic significance in all three groups (data not shown), although a larger sample size is required for validation.

We also demonstrated that the co-expression of NPM and TFF3 predicted the poorest prognosis (Fig. 2D). Recent studies also demonstrated that both NPM and TFF3 are involved in the regulation of apoptosis via the $\mathrm{NF}-\kappa \mathrm{B}$ pathway $(7,8,19)$. Tobita et al (29) reported that E2F1, which is implicated in hepatocarcinogenesis, may increase the expression of TFF3 by upregulating DNA-binding protein A, which is associated with advanced stages of human hepatocellular carcinoma. In addition, the activation of E2F1 is regulated by NPM/B23 via modulating the promoter binding of NF- $\kappa \mathrm{B}(8)$. These findings, taken together with ours, suggest that NPM and TFF3 may be involved in the occurrence or development of GC, possibly through interacting with each other in the $\mathrm{NF}-\kappa \mathrm{B}$ pathway. Therefore, the co-detection of NPM and TFF3 may serve as a more predictive index for GC patients. However, whether NPM and TFF3 act independently or cooperatively to increase the malignant potential of GC requires further investigation. In the present study, the correlation analysis identified no significant correlation between the protein level of NPM and TFF3 ( $r=0.11119, P=0.2520$, Table II). In the future, further studies on NPM and TFF3, possibly at the nucleic acid level, should be conducted to elucidate whether there is an interaction, as well as the type of that interaction between these two factors in GC.

In conclusion, the present study provided direct evidence that NPM is an independent prognostic factor in postoperative GC patients. We also confirmed that TFF3 expression is associated with poor prognosis. Of note, the co-expression of NPM and TFF3 proved to be an independent prognostic factor in postoperative GC patients. The combined detection of the co-expression of the two factors may serve as a more predictive index of GC patient prognosis. However, the molecular mechanism underlying the association of NPM and TFF3 requires elucidation by further studies.

\section{Acknowledgements}

This study was supported by the Shandong Natural Science Foundation (grant no. 2009HW024), the Shandong Excellent Young Scientist Research Award Fund Project (grant nos. 2006BSB14114 and BS2010YY013) and the Shandong Tackle Key Problems in Science and Technology (grant no. 2010GSF10245).

\section{References}

1. He W, Tang B, Yang D, et al: Double-positive expression of high-mobility group box 1 and vascular endothelial growth factor $\mathrm{C}$ indicates a poorer prognosis in gastric cancer patients. World J Surg Oncol 11: 161, 2013.

2. Zhang YZ, Zhang LH, Gao Y, et al: Discovery and validation of prognostic markers in gastric cancer by genome-wide expression profiling. World J Gastroenterol 17: 1710-1717, 2011.

3. Jia YF, Xiao DJ, Ma XL, et al: Differentiated embryonic chondrocyte-expressed gene 1 is associated with hypoxia-inducible factor 1alpha and Ki67 in human gastric cancer. Diagn Pathol 8: 37, 2013.

4. Jin J, Jin T, Quan M, Piao Y and Lin Z: Ezrin overexpression predicts the poor prognosis of gastric adenocarcinoma. Diagn Pathol 7: 135, 2012.

5. Sotoudeh K, Hashemi F, Madjd Z, Sadeghipour A, Molanaei S and Kalantary E: The clinicopathologic association of c-MET overexpression in Iranian gastric carcinomas; an immunohistochemical study of tissue microarrays. Diagn Pathol 7: 57, 2012.

6. Grisendi S, Mecucci C, Falini B and Pandolfi PP: Nucleophosmin and cancer. Nat Rev Cancer 6: 493-505, 2006.

7. Khandelwal N, Simpson J, Taylor G, Rafique S, Whitehouse A, Hiscox J and Stark LA: Nucleolar NF-kappaB/RelA mediates apoptosis by causing cytoplasmic relocalization of nucleophosmin. Cell Death Differ 18: 1889-1903, 2011.

8. Lin CY, Liang YC and Yung BY: Nucleophosmin/B23 regulates transcriptional activation of $\mathrm{E} 2 \mathrm{~F} 1$ via modulating the promoter binding of NF-kappaB,E2F1 and pRB. Cell Signal 18: 2041-2048, 2006.

9. Nozawa Y, Van Belzen N, Van der Made AC, Dinjens WN and Bosman FT: Expression of nucleophosmin/B23 in normal and neoplastic colorectal mucosa. J Pathol 178: 48-52, 1996.

10. Yun JP, Miao J, Chen GG, et al: Increased expression of nucleophosmin/B23 in hepatocellular carcinoma and correlation with clinicopathological parameters. Br J Cancer 96: 477-484, 2007.

11. Subong EN, Shue MJ, Epstein JI, Briggman JV, Chan PK and Partin AW: Monoclonal antibody to prostate cancer nuclear matrix protein (PRO:4-216) recognizes nucleophosmin/B23. Prostate 39: 298-304, 1999.

12. Tsui KH, Cheng AJ, Chang Pe, Pan TL and Yung BY: Association of nucleophosmin/B23 mRNA expression with clinical outcome in patients with bladder carcinoma. Urology 64: 839-844, 2004.

13. Zhang Y: The ARF-B23 connection: implications for growth control and cancer treatment. Cell Cycle 3: 259-262, 2004.

14. Mascaux C, Bex F, Martin B, et al: The role of NPM, p14arf and MDM2 in precursors of bronchial squamous cell carcinoma. Eur Respir J 32: 678-686, 2008.

15. Tanaka M, Sasaki H, Kino I, Sugimura T and Terada M: Genes preferentially expressed in embryo stomach are predominantly expressed in gastric cancer. Cancer Res 52: 3372-3377, 1992.

16. Muskett FW, May FE, Westley BR and Feeney J: Solution structure of the disulfide-linked dimer of human intestinal trefoil factor (TFF3): the intermolecular orientation and interactions are markedly different from those of other dimeric trefoil proteins. Biochemistry 42: 15139-15147, 2003.

17. Katoh M: Trefoil factors and human gastric cancer (Review). Int J Mol Med 12: 3-9, 2003.

18. Meyer zum Buschenfelde D, Hoschutzky H, Tauber R and Huber O: Molecular mechanisms involved in TFF3 peptide-mediated modulation of the E-cadherin/catenin cell adhesion complex. Peptides 25: 873-883, 2004.

19. Chen YH, Lu Y, De Plaen IG, Wang LY and Tan XD: Transcription factor NF-kappaB signals antianoikic function of trefoil factor 3 on intestinal epithelial cells. Biochem Biophys Res Commun 274: 576-582, 2000.

20. Leung WK, Yu J, Chan FK, et al: Expression of trefoil peptides (TFF1, TFF2, and TFF3) in gastric carcinomas, intestinal metaplasia, and non-neoplastic gastric tissues. J Pathol 197: 582-588, 2002.

21. Yamachika T, Werther JL, Bodian C, et al: Intestinal trefoil factor: a marker of poor prognosis in gastric carcinoma. Clin Cancer Res 8: 1092-1099, 2002.

22. Zhou F, Qiu W, Sun L, et al: Clinical significance of nucleophosmin/B23 and human epidermal growth factor receptor $2 /$ neu expressions in gastric cancers. APMIS 121: 582-591, 2013.

23. Xu CC, Yue L, Wei HJ, et al: Significance of TFF3 protein and Her-2/neu status in patients with gastric adenocarcinoma. Pathol Res Pract 209: 479-485, 2013. 
24. Xu D, Huang Y, Geng Q, Guan Y, Li Y, et al: Effect of lymph node number on survival of patients with lymph node-negative gastric cancer according to the 7th edition UICC TNM system. PLoS One 7: e38681, 2012.

25. Ding A, Zhao W, Shi X, et al: Impact of NPM, TFF3 and TACC1 on the prognosis of patients with primary gastric cancer. PLoS One 8: e82136, 2013.

26. Tsui KH, Juang HH, Lee TH, Chang PL, Chen CL and Yung BY: Association of nucleophosmin/B23 with bladder cancer recurrence based on immunohistochemical assessment in clinical samples. Acta Pharmacol Sin 29: 364-370, 2008.
27. Meng JR, Tang HZ, Zhou KZ, Shen WH and Guo HY: TFF3 and survivin expressions associate with a lower survival rate in gastric cancer. Clin Exp Med 13: 297-303, 2013.

28. Dhar DK, Wang TC, Tabara H, et al: Expression of trefoil factor family members correlates with patient prognosis and neoangiogenesis. Clin Cancer Res 11: 6472-6478, 2005.

29. Tobita H, Kajino K, Inami K, et al: Gene expression profile of DNA binding protein A transgenic mice. Int J Oncol 29: 673-679, 2006. 